\title{
Persistent Hijacking of Brain Proteasomes in HIV-Associated Dementia
}

Trung P. Nguyen, ${ }^{*}$ Vicki M. Soukup, ${ }^{\dagger}$ and Benjamin B. Gelman*

From the Departments of Pathology* and Neurology, ${ }^{\dagger}$ the University of Texas Medical Branch, Galveston, Texas

Immunoproteasome induction sustains class 1 antigen presentation and immunological vigilance against HIV-1 in the brain. Investigation of HIV-1-associated alterations in brain protein turnover by the ubiquitinproteasome system was performed by (1) determining proteasome subunit changes associated with persistent brain inflammation due to HIV-1; (2) determining whether these changes are related to HIV-1 neurocognitive disturbances, encephalitis, and viral loads; and (3) localizing proteasome subunits in brain cells and synapses. On the basis of neurocognitive performance, virological, and immunological measurements obtained within 6 months before death, 153 autopsy cases were selected. Semiquantitative immunoblot analysis performed in the dorsolateral prefrontal cortex revealed up to threefold induction of immunoproteasome subunits LMP7 and PA28 $\alpha$ in HIV-1-infected subjects and was strongly related to diagnoses of neuropsychological impairment and HIV encephalitis. Low performance on neurocognitive tests specific for dorsolateral prefrontal cortex functioning domains was selectively correlated with immunoproteasome induction. Immunohistochemistry and laser confocal microscopy were then used to localize immunoproteasome subunits to glial and neuronal elements including perikarya, dystrophic axons, and synapses. In addition, HIV loads in brain tissue, cerebrospinal fluid, and blood plasma were robustly correlated to immunoproteasome levels. This persistent "hijacking" of the proteasome by HIV-1mediated inflammatory response and immunoproteasome induction in the brain is hypothesized to impede turnover of folded proteins in brain cells. This would disrupt neuronal and synaptic protein dynamics, contributing to HIV-1 neurocognitive disturbances. (Am J Patbol 2010, 176:893-902; DOI: 10.2353/ajpath.2010.090390)

People infected with HIV-1 are vulnerable to syndromes of neurocognitive impairment at a relatively young age, including HIV-associated dementia (HAD) and mild cognitive and motor disturbance (MCMD). Highly active antiretroviral therapy suppresses HIV-1 replication, prevents dementia, and prolongs survival, but does not eradicate HIV-1 infection. ${ }^{1}$ Inflammation is the putative driving force behind MCMD and HAD. ${ }^{2,3}$ HIV-1 enters the central nervous system (CNS) via infected macrophages and triggers inflammatory changes including the release of cytokines, neurotoxins, and toxic viral proteins. HIV-1 produces inflammatory changes neuropathologically that are known as HIV encephalitis (HIVE). ${ }^{4}$ HIVE and HAD are correlated with each other, which supports a proinflammatory mechanism for the pathophysiology of dementia in many, but not all cases. ${ }^{5}$

Inflammation has an influence on protein turnover through the ubiquitin proteasome system (UPS). ${ }^{6-8}$ The proteasome is a multicatalytic proteinase that is the main route of cellular protein degradation and turnover. ${ }^{9}$ Inflammatory mediators including interferon- $\gamma(\mathrm{IFN}-\gamma)$ and tumor necrosis factor $\alpha$ modify expression of proteasome subunits to promote the synthesis of the immunoproteasome complex (IPS). ${ }^{6-8,10-15}$ This causes switching from the synthesis of "standard" constitutive proteasome complexes (CPS), which process folded proteins through the UPS, to IPS complexes, which are specialized for processing unfolded polypeptides for class 1 antigen presentation in viral defense. ${ }^{10,15}$ The "borrowing" of the UPS by IPS induction is not pathological to cells because it subsides quickly after an infected host eradicates the pathogen. ${ }^{7}$ Eradication of HIV-1 in the CNS, however, is not achieved and a vigilant immune defense must be maintained. ${ }^{15-17}$ This persistent inflammatory drive in HIVIAIDS could exert a potentially harmful slowing of protein turnover through the UPS. That in turn could have a profound influence in the CNS because impairment of protein turnover interferes with synaptic function and impairs learning and memory formation. ${ }^{18,19}$ A persistent slowing of protein turnover via the UPS probably leads to

Supported by grants R01 MH69200 and U01-MH083507 from the National Institutes of Health.

Accepted for publication October 20, 2009.

Address reprint requests to Benjamin B. Gelman, M.D., Ph.D., the University of Texas Medical Branch, Department of Pathology, 301 University Blvd, Route 0609, Galveston, TX 77555-0609. E-mail: bgelman@utmb.edu. 
Table 1. Immunoblotting Primary Antibodies

\begin{tabular}{|c|c|c|c|c|}
\hline Antibody (clone) & Source & ID & Type & Dilution \\
\hline \multicolumn{5}{|l|}{$20 S \alpha$-type } \\
\hline$\alpha 4$ (МСР79) & Biomol & PW9140 & Mouse mAb & $1: 1000$ \\
\hline$\alpha 6$ (MCP20) & Biomol & PW8100 & Mouse mAb & $1: 1000$ \\
\hline$\alpha 7$ (MCP72) & Biomol & PW8110 & Mouse mAb & $1: 1000$ \\
\hline \multicolumn{5}{|l|}{$20 \mathrm{~S} \beta$-type } \\
\hline $\mathrm{X} / \beta 5$ & Affinity Bioreagents & PA1-977 & Rabbit pAb & $1: 1000$ \\
\hline$Y / \beta 1$ & Affinity Bioreagents & PA1-978 & Rabbit pAb & $1: 1000$ \\
\hline$Z / \beta 2$ & Affinity Bioreagents & PA1-979 & Rabbit pAb & $1: 1000$ \\
\hline LMP7/ß5i (LMP7-1) & Biomol & PW8845 & Mouse mAb & $1: 1000$ \\
\hline 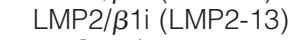 & Biomol & PW8840 & Mouse mAb & $1: 1000$ \\
\hline 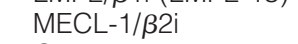 & Biomol & PW8150 & Rabbit pAb & $1: 1000$ \\
\hline \multicolumn{5}{|l|}{ 19S regulator ATPase } \\
\hline Rpt1 & Biomol & PW8315 & Rabbit pAb & $1: 1000$ \\
\hline Rpt5 & Affinity Bioreagents & PA1-967 & Rabbit pAb & $1: 500$ \\
\hline \multicolumn{5}{|c|}{ 19S regulator non-ATPase } \\
\hline Rpn2 & Affinity Bioreagents & PA1-973 & Rabbit pAb & $1: 1000$ \\
\hline Rpn8 & Affinity Bioreagents & PA1-1963 & Rabbit pAb & $1: 1000$ \\
\hline \multicolumn{5}{|l|}{$11 S$ regulator } \\
\hline PA28 $\alpha$ & Biomol & PW8185 & Rabbit pAb & $1: 1000$ \\
\hline
\end{tabular}

mAb, monoclonal antibody; pAb, polyclonal antibody.

accumulation of misfolded ubiquitinylated proteins in pathological aging, which is a hallmark neuropathological change in neurodegenerative diseases. ${ }^{20-27} \mathrm{An}$ increase in ubiquitin-protein conjugates was reported in HIV/AIDS brains that was associated with inflammation and altered synaptic protein content. ${ }^{28}$ Here we report that HIV-1 infection exerts a strong influence on brain UPS that is associated with neurocognitive impairment and neuropathological changes.

\section{Materials and Methods}

\section{Study Subjects}

Eighty-eight HIV-positive $\left(\mathrm{HIV}^{+}\right)$subjects were selected from the National NeuroAIDS Tissue Consortium ${ }^{29}$ and/or the Texas NeuroAIDS Research Center. Forty-seven $\mathrm{HIV}^{+}$subjects had neuropsychological impairment (NPI), including 23 subjects with HAD and 24 subjects with MCMD. Eleven $\mathrm{HIV}^{+}$subjects did not have syndromic impairment. Twenty $\mathrm{HIV}^{+}$subjects had NPI combined with other conditions (NPI-O), which precluded a diagnosis of HAD or MCMD. Ten HIV ${ }^{+}$decedents were included that did not have neurocognitive diagnoses. Twenty subjects had HIVE. All HIV ${ }^{+}$patients were treated with antiretroviral therapy. Sixty-five HIV-negative $\left(\mathrm{HIV}^{-}\right)$ subjects of comparable age, gender, and race with no significant neuropathological findings were included. The protection of human subjects was approved by the institutional review board of the University of Texas Medical Branch at Galveston under protocol 98-402.

\section{Brain Tissue Preparation and Western Blots}

Samples from the dorsolateral prefrontal cortex (DLPFC) and frontal white matter (WM) from fresh-frozen brain slices stored at $-80^{\circ} \mathrm{C}$ were homogenized by silica bead beating and sonication in $10 \mathrm{mmol} / \mathrm{L}$ Tris- $\mathrm{HCl}, 0.5 \mathrm{mmol} / \mathrm{L}$
Dithiothreitol, $0.03 \%$ Triton $\mathrm{X}-100,5 \mathrm{mmol} / \mathrm{L} \mathrm{MgCl}_{2}$, and $\mathrm{pH}$ 7.8. Homogenates (10 to $30 \mu \mathrm{g}$ total protein) were added to 2X Laemmli Sample Buffer (Bio-Rad Laboratories, Hercules, CA) with $5 \% \beta$-mercaptoethanol, boiled, and loaded into Criterion Precast Tris-HCL gels (Bio-Rad Laboratories) for SDS-polyacrylamide gel electrophoresis. Protein was transferred to polyvinylidene difluoride membranes. The membranes were then blocked with 5\% nonfat dry milk. Primary antibodies from Biomol International, Inc. (Plymouth Meeting, PA) and Affinity Bioreagents (Golden, CO) (Table 1), anti-rabbit or anti-mouse secondary antibodies and Enhanced Chemiluminescence Detection Reagent (Amersham Biosciences, Piscataway, NJ), were applied. Exposed X-ray film band densities were quantified with One-Dscan (BD Biosciences Bioimaging, Rockville, MD).

\section{Neurocognitive Testing}

A battery of tests was designed by the National NeuroAIDS Tissue Consortium to evaluate domains of cognitive functioning in MCMD and HAD. ${ }^{29,30}$ The Wisconsin Card Sorting Test-64 (WCST-64) assesses abstract and executive functioning driven primarily by frontal lobe circuitry. The Wechsler Adult Intelligence Scale III subtests, Digit Symbol and Symbol Search, provide an index of information processing speed. Also included were the Hopkins Verbal Learning Test Revised, the Brief Visuospatial Memory Test Revised, the Paced Auditory Serial Addition Test, the F-A-S Test, and the Wide Range Assessment Test. A neuropsychologist rendered the neuropsychological diagnosis.

\section{Viral Load}

Laboratory values of plasma CD4 ${ }^{+}$lymphocyte count and viral loads (VL) in plasma and cerebrospinal fluid (CSF) were obtained within 6 months of death. Plasma 
Table 2. Immunofluorescence Primary Antibodies

\begin{tabular}{|c|c|c|c|c|}
\hline Antibody (Clone) & Source & ID & Type & Dilution \\
\hline \multicolumn{5}{|l|}{ Immunoproteasome } \\
\hline LMP2/31i & Abcam Cambridge, MA & ab3328 & Rabbit pAb & $1: 1000$ \\
\hline PA28 $\alpha$ & Biomol & PW8185 & Rabbit pAb & $1: 1000$ \\
\hline \multicolumn{5}{|l|}{ Neurons } \\
\hline NeuN (A60) & Millipore Billerica, MA & MAB377 & Mouse mAb & $1: 100$ \\
\hline Neurofilament (SPM204) & Abcam & $a b 17832$ & Mouse mAb & $1: 50$ \\
\hline Synaptophysin (SVP-38) & Sigma Aldrich & S5768 & Mouse mAb & $1: 200$ \\
\hline \multicolumn{5}{|l|}{ Astrocytes } \\
\hline $\begin{array}{l}\text { GFAP (GA5) } \\
\text { Oligodendrocytes }\end{array}$ & Millipore & MAB360 & Mouse mAb & $1: 400$ \\
\hline OMG (4A9) & Lifespan Seattle, WA & LS-C27282 & Mouse mAb & $1: 300$ \\
\hline \multicolumn{5}{|l|}{ Macrophage/microglia } \\
\hline CD68 (KP1) & Dako & M0814 & Mouse mAb & $1: 100$ \\
\hline
\end{tabular}

mAb, monoclonal antibody; pAb, polyclonal antibody.

and CSF were analyzed by using the Roche AMPLICOR HIV-1 Monitor Test (Branchburg, NJ). Brain tissue RNA was extracted by using the RNeasy Lipid Tissue Mini Kit (Qiagen, Valencia, CA) for HIV-1 RNA single copy detection as described by Palmer et al. ${ }^{31}$ One microgram of brain RNA and $1 \mu \mathrm{mol} / \mathrm{L}$ of antisense primer 84R were used in $20 \mu \mathrm{l}$ reaction (iScript cDNA Synthesis Kit, Bio-Rad Laboratories, Hercules, CA). Four microliters of cDNA was used for $25 \mu \mathrm{l}$ real-time PCR by using JumpStart Taq ReadyMix for Quantitative PCR (Sigma Aldrich, St. Louis, MO) and SmartCycler (Cepheid, Sunnyvale, CA). Results were standardized against a known brain secondary standard.

\section{Microscopy}

Immunoperoxidase histochemistry was performed on formalin-fixed paraffin-embedded tissue sections that were quenched with $3 \%$ hydrogen peroxide in methanol, irradiated with microwaves in sodium citrate buffer (10 $\mathrm{mmol} / \mathrm{L}$ sodium citrate, $0.05 \%$ Tween-20, $\mathrm{pH} 6.0$ ), and blocked with $0.1 \%$ nonfat dry milk and $1 \%$ normal goat serum. Rabbit polyclonal anti-LMP7 (1:1000) or antiPA28 $\alpha$ (1:1000) (BIOMOL), secondary antibody, and Vectastain avidin-biotin complex and Peroxidase substrate diaminobenzidine kits (Vector Laboratories, Burlingame, CA) were applied. Immunohistochemistry for $\mathrm{CD}^{+}$lymphocytes was similarly performed. Antigen retrieval was performed in $1 \mathrm{mmol} / \mathrm{L}$ EDTA, $\mathrm{pH}$ 8.0. Mouse anti-CD8 (clone 4B11, 1:40) (Novocastra Labs, Newcastle on Tyne, UK) was applied, followed by the labeled streptavidin-biotin method and diaminobenzidine staining (Dako, Glostrup, Denmark). Slides were then counterstained with Harris Hematoxylin (Fisher Scientific, Pittsburgh, PA). For immunofluorescence, sections were steamed in sodium citrate buffer and blocked with Image-iT FX signal enhancer (Invitrogen Molecular Probes, Eugene, OR), $5 \%$ bovine serum albumin, and $5 \%$ normal goat serum. Primary antibodies (Table 2) and AlexaFluor fluorochrome-conjugated secondary antibodies (Invitrogen Molecular Probes) were applied. Autofluorescence was quenched with $1 \%$ Sudan Black B (Sigma Aldrich) in $70 \%$ ethanol. Coverslips were mounted with Slow Fade Gold with 4',6-diamidino-2-phenylindole (Invitrogen Molecular Probes). Confocal images of single optical sections of 500-nm thickness were acquired with a Zeiss LSM 510 UV META laser scanning confocal microscope consisting of an Axiovert 200M Inverted Microscope equipped with Argon, dual HeNe, and UV lasers, fluorescence filters set for DAPI, fluorescein isothiocyanate, and tetramethylrhodamine isothicyanate, a scanning module with visible and UV acousto optical tunable filters, two independent photomultiplier tubes (PMT) array (Carl Zeiss Microlmaging, Inc, Thornwood, NY).

\section{Statistics}

SAS/STAT 9.1.3 PROC REG (SAS Institute, Inc, Cary, NC), Microsoft Excel 2003 (Microsoft Corporation, Redmond, WA), and GraphPad InStat 3.06 (GraphPad Software, Inc, La Jolla, CA) were used. Analysis of variance was used for comparisons between more than two groups. Data with normal distribution were analyzed by using one-way analysis of variance followed by the Tukey-Kramer Multiple Comparisons Test, or Student's $t$ test. Otherwise, the Kruskal-Wallis Test followed by Dunn's Multiple Comparison Test was used. Error bars represent SEM. Regression analysis was used to determine effects of age and HIV status.

\section{Results}

\section{Proteasome and Immunoproteasome Subunits}

Initial immunoblot screening compared eight $\mathrm{HIV}^{-}$subjects and eight $\mathrm{HIV}^{+}$subjects with HIVE and/or neurocognitive impairment to identify proteasome subunits with altered protein levels for further analysis. Screening results showed that levels of IPS 20S $\beta$ subunits LMP2, LMP7, and MECL-1 were increased substantially in DLPFC and WM of $\mathrm{HIV}^{+}$subjects compared with the basal IPS levels in $\mathrm{HIV}^{-}$subjects (Figure 1B). PA28 $\alpha$, an inducible subunit of the $11 \mathrm{~S}$ regulatory complex, was also increased in $\mathrm{HIV}^{+}$subjects (Figure 1D). CPS 20S $\beta$ (Figure $1 \mathrm{~A})$ and $\alpha$ (Figure $1 \mathrm{E}$ ) subunits were not changed. The majority of CPS 19S subunits screened were unchanged (Figure 1C). However, one delta 19S nonATPase subunit known as Rpn2, a likely chaperonin, was decreased in DLPFC of $\mathrm{HIV}^{+}$subjects. 


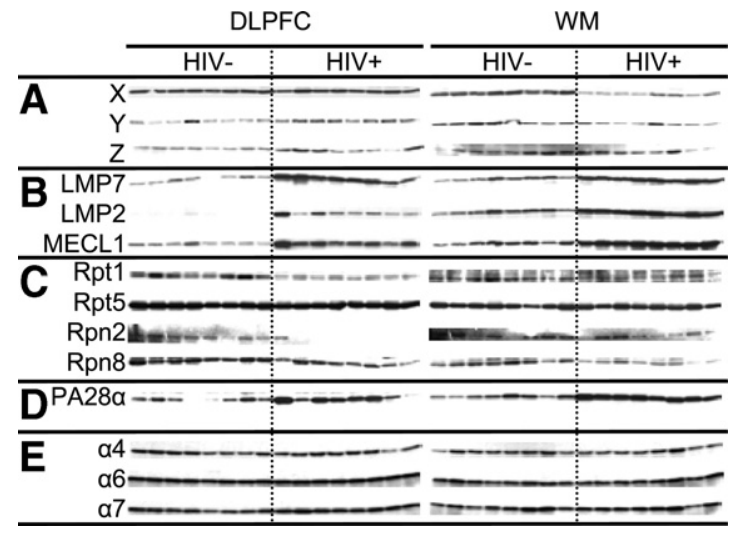

Figure 1. Western blots illustrate altered proteasome subunit concentrations in DLPFC and WM from eight $\mathrm{HIV}^{+}$subjects with HIVE and/or neurocognitive impairment compared with eight $\mathrm{HIV}^{-}$subjects. A: Constitutive $20 \mathrm{~S}$ proteasome subunits $\mathrm{X}(\beta 5), \mathrm{Y}(\beta 1)$, and $\mathrm{Z}(\beta 2)$ were not changed in $\mathrm{HIV}^{+}$ subjects in DLPFC or WM. B: Three inducible 20S immunoproteasome $\beta$ subunits were increased in DLPFC and WM of $\mathrm{HIV}^{+}$subjects. C: The majority of constitutively expressed $19 \mathrm{~S}$ proteasome regulatory complex subunits remained unchanged, except for Rpn2, which showed a decrease in the DLPFC of $\mathrm{HIV}^{+}$subjects. D: The immunoproteasome $11 \mathrm{~S}$ regulatory subunit PA28 $\alpha$ was increased in DLPFC and WM of HIV ${ }^{+}$subjects. E: 20 S $\alpha$ subunits were not changed.

\section{$\mathrm{CD8}^{+}$Lymphocytes}

In our screening panel, we explored whether $\mathrm{CD} 8^{+}$lymphocytes, which can produce IFN- $\gamma$, might be more prevalent with IPS induction. Histologically stained $\mathrm{CD} 8^{+}$lymphocytes were unexpectedly less prevalent in subjects with IPS induction compared with those without IPS induction (13.73 $\pm 12.7 \mathrm{cells} / \mathrm{cm}^{2}$ versus $35.9 \pm 20.8$ cells/ $\mathrm{cm}^{2}, \mathrm{df}=15, P=0.025$, not illustrated).

\section{Association with Neuropathology and Neuropsychological Diagnoses}

LMP7 (Figure 2, A and B) and PA28 $\alpha$ (Figure 2, C and D) were measured in DLPFC and WM samples from all subjects by using serial Western blotting with loading controls and densitometrical analysis. The average levels of these inducible subunits in $\mathrm{HIV}^{+}$subjects were significantly increased approximately twofold to threefold compared with $\mathrm{HIV}^{-}$subjects.

LMP7 (Figure 3, A and B) and PA28 $\alpha$ (Figure 3, C and D) were significantly increased in those subjects with HIVE in DLPFC and WM. The average increase in HIVE was three- to fivefold compared with $\mathrm{HIV}^{-}$subjects. In $\mathrm{HIV}^{+}$subjects without HIVE, a more modest yet significant increase was present that was approximately twofold higher than $\mathrm{HIV}^{-}$subjects.

Subjects with NPI had significantly increased LMP7 (Figure 4, A and B) and PA28 $\alpha$ (Figure 4, C and D) in DLPFC and WM. Average increases were approximately two- to fourfold, with the most pronounced affect seen with WM PA28 $\alpha$ measurements. $\mathrm{HIV}^{+}$subjects without NPI had little or no increase in either inducible subunit. $\mathrm{HIV}^{+} \mathrm{NPI}-\mathrm{O}$ subjects also had significantly increased IPS subunits, but the increase was less pronounced than those with NPI.
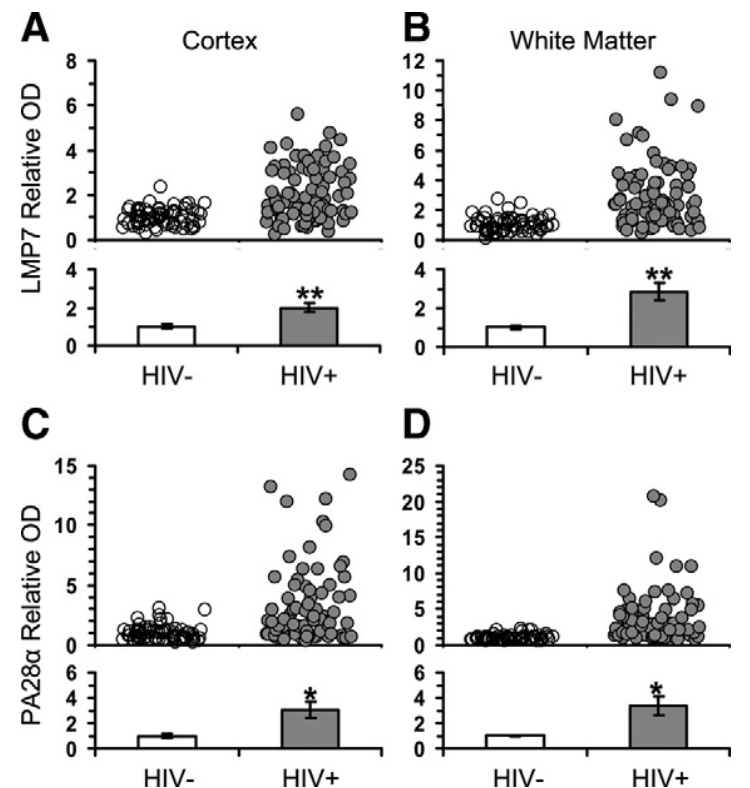

D

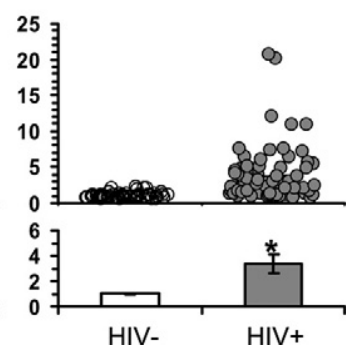

Figure 2. Immunoproteasome subunit proteins in $88 \mathrm{HIV}^{+}$and $65 \mathrm{HIV}^{-}$subjects were quantified by using densitometry of calibrated Western blots with loading controls. Averaged LMP7 was increased 99\% in DLPFC (A) and 184\% in WM (B). PA28 $\alpha$ was increased by $204 \%$ in DLPFC (C) and $233 \%$ in WM (D). Statistics: Student's $t$ test; ${ }^{*} P<10^{-7} ;{ }^{* *} P<10^{-10}$. OD $=$ optical density.

The decrease in DLPFC constitutive 19S regulatory complex subunit Rpn2 seen in proteasome subunit screening was investigated. A slight decrease in $\mathrm{HIV}^{+}$ subjects was not significant (not shown). Further analysis
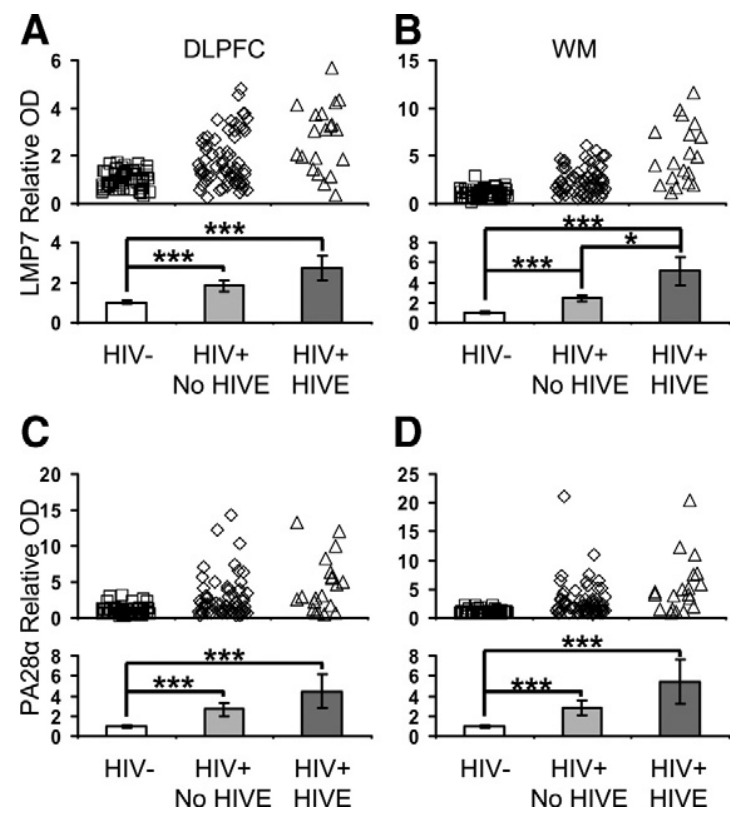

Figure 3. Significantly increased immunoproteasome subunit LMP7 and PA28 $\alpha$ concentration in $\mathrm{HIV}^{+}$subjects with and without HIVE. A: DLPFC LMP7 levels in $\mathrm{HIV}^{+}$subjects with and without HIVE were increased $173 \%$ and $81 \%$, respectively, compared with $\mathrm{HIV}^{-}$subjects. B: WM LMP7 in HIVE was increased $414 \%$ and $114 \%$ compared with $\mathrm{HIV}^{-}$subjects and $\mathrm{HIV}^{+}$ subjects without HIVE, respectively. LMP7 levels of $\mathrm{HIV}^{+}$subjects without HIVE were $140 \%$ greater than $\mathrm{HIV}^{-}$subjects. C: DLPFC PA28 $\alpha$ levels of $\mathrm{HIV}^{+}$ subjects with and without HIVE were 349\% and 166\% higher than $\mathrm{HIV}^{-}$ subjects, respectively. D: In WM, PA28 $\alpha$ was increased $443 \%$ and $185 \%$ in $\mathrm{HIV}^{+}$subjects with and without HIVE, respectively, compared with HIV subjects. Statistics: one-way analysis of variance with Tukey-Kramer Multiple Comparisons Test; ${ }^{*} P<0.05$; ${ }^{* * * * *} P<0.001$. OD $=$ optical density. 

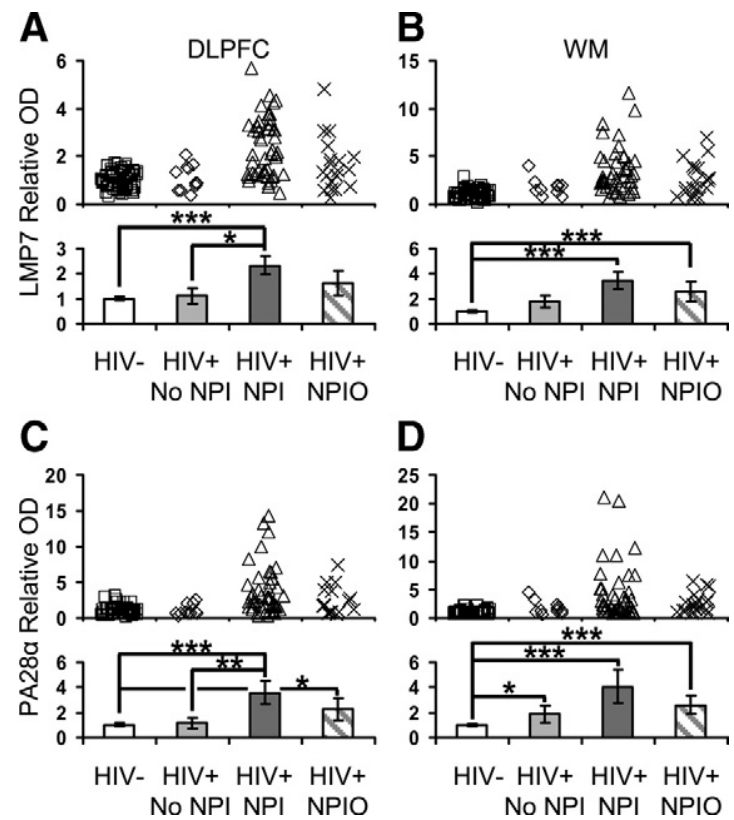

HIV- HIV+ HIV+ HIV+ No NPI NPI NPIO

Figure 4. Increased immunoproteasome subunit LMP7 and PA28 $\alpha$ concentration significantly related to NPI. Subunit level increases in $\mathrm{HIV}^{+}$subjects with NPI-O were less pronounced. No substantial change was observed with $\mathrm{HIV}^{+}$subjects without NPI. A: DLPFC LMP7 levels in $\mathrm{HIV}^{+}$NPI subjects were increased $133 \%$ and $110 \%$ compared with $\mathrm{HIV}^{-}$subjects and $\mathrm{HIV}^{+}$subjects without NPI, respectively. B: WM LMP7 increased 243\% in those with NPI and $154 \%$ in those with NPI-O compared with HIV ${ }^{-}$subjects. C: DLPFC PA28 $\alpha$ in $\mathrm{HIV}^{+}$NPI subjects was increased $259 \%$ compared with $\mathrm{HIV}^{-}$subjects and $216 \%$ compared with HIV $^{+}$subjects without NPI. HIV ${ }^{+}$NPI-O subjects had a $126 \%$ increase compared with $\mathrm{HIV}^{-}$subjects. D: WM PA28 $\alpha$ was increased $85 \%$ in $\mathrm{HIV}^{+}$subjects without NPI, 305\% in $\mathrm{HIV}^{+}$NPI subjects, and $159 \%$ in $\mathrm{HIV}^{+}$NPI-O subjects compared with $\mathrm{HIV}^{-}$subjects. Statistics: one-way analysis of variance with Tukey-Kramer Multiple Comparisons Test; ${ }^{*} P<$ $0.05 ;{ }^{* *} P<0.01 ;{ }^{* * * *} P<0.001$. OD $=$ optical density.

revealed mild decreases in Rpn2 associated with NPI and HIVE (not shown) that may account for the decrease seen in screening, but with the accompanying data of comparable 19S subunits, provided only minimal evidence for alteration in the constitutively expressed 19S regulatory complex.

\section{Effects of Age}

Regression analysis was used to determine the effect of age on immunoproteasome subunit concentrations in $\mathrm{HIV}^{+}$and $\mathrm{HIV}^{-}$subjects (Table 3). DLPFC PA28 $\alpha$ and LMP7 regressions with age were equivalent between

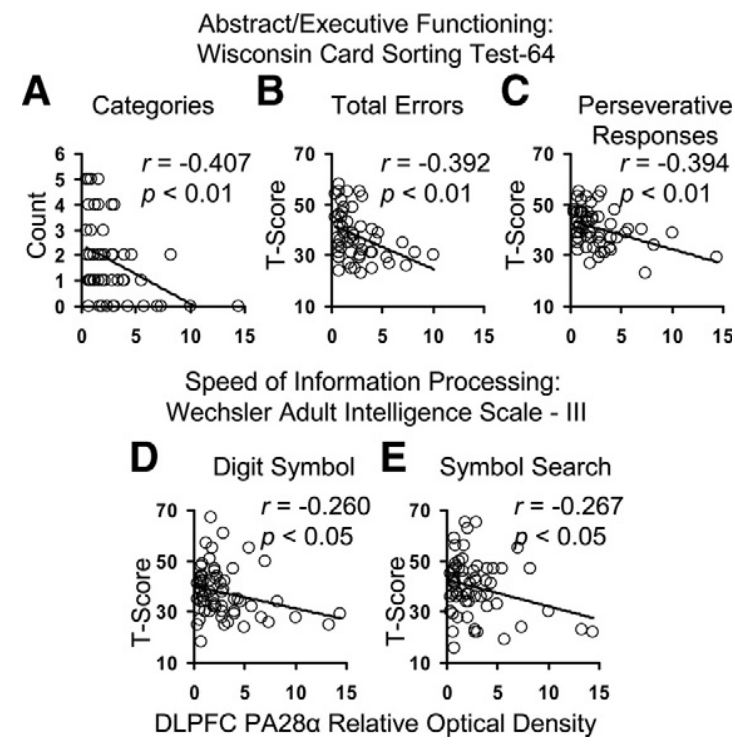

Figure 5. Immunoproteasome induction in DLPFC, but not WM, was correlated with worse performance in the abstract and executive functioning and speed of information processing neurocognitive domains. Decreased performance on the WCST-64 reflects abstract and executive function, and it was correlated with PA28 $\alpha$ (A-C) and LMP7 (not illustrated). Scores for (A) categories completed, $(\mathbf{B})$ total errors made, and $(\mathbf{C})$ perseverative responses are shown. PA28 $\alpha$, but not LMP7, also was correlated with decreased performance on the Wechsler Adult Intelligence Scale III (WAIS-III) scores for (D) Digit-Symbol and (E) Symbol Search subtests, which reflects a decrease in the speed of information processing. Other neurocognitive domains that were tested were not correlated significantly. Statistics: Pearson's correlation.

$\mathrm{HIV}^{-}$and $\mathrm{HIV}^{+}$subjects and not significantly different from zero, except for LMP7 in $\mathrm{HIV}^{-}$subjects. White matter regressions were unequal between $\mathrm{HIV}^{+}$and $\mathrm{HIV}^{-}$ subjects for PA28 and LMP7, with significant negative regression with age in $\mathrm{HIV}^{+}$subjects and no significant regressions for $\mathrm{HIV}^{-}$subjects.

\section{Correlation with Neurocognitive Test Performance}

DLPFC PA28 $\alpha$ (Figure 5, A-C) and LMP7 (not illustrated) were negatively correlated with WCST-64 scores, which are mediated in the DLPFC ${ }^{32,33}$ and reflect impairment of abstract and executive functioning. This relationship was specific to the DLPFC and was not evident for WM (not illustrated). DLPFC PA28 $\alpha$ was also negatively correlated with Wechsler Adult Intelligence Scale III subtests includ-

Table 3. Aging and Proteasome Subunit Levels Regression Analysis Using Log-transformed Values

\begin{tabular}{|c|c|c|c|c|c|}
\hline \multirow[b]{2}{*}{ Subunit } & \multirow[b]{2}{*}{ Status } & \multirow[b]{2}{*}{ Slope } & \multicolumn{2}{|c|}{ Interaction with age } & \multirow{2}{*}{$\frac{\mathrm{HIV}^{-} \text {vs. } \mathrm{HIV}^{+}}{P}$} \\
\hline & & & SE & $P$ & \\
\hline \multicolumn{6}{|c|}{ Dorsolateral prefrontal cortex } \\
\hline \multirow[t]{2}{*}{ LMP7 } & $\mathrm{HIV}^{+}$ & -0.0036 & 0.0064 & NS & NS \\
\hline & $\mathrm{HIV}^{-}$ & 0.0108 & 0.0041 & $<0.01$ & \\
\hline \multirow[t]{2}{*}{ PA28 $\alpha$} & $\mathrm{HIV}^{+}$ & -0.0081 & 0.0092 & NS & NS \\
\hline & $\mathrm{HIV}^{-}$ & 0.0056 & 0.0059 & NS & \\
\hline \multicolumn{6}{|l|}{ White matter } \\
\hline \multirow[t]{2}{*}{ LMP7 } & $\mathrm{HIV}^{+}$ & -0.0250 & 0.0070 & $<0.001$ & $<0.01$ \\
\hline & $\mathrm{HIV}^{-}$ & -0.0016 & 0.0046 & NS & \\
\hline \multirow[t]{2}{*}{ PA28 $\alpha$} & $\mathrm{HIV}^{+}$ & -0.0306 & 0.0073 & $<0.0001$ & $<0.001$ \\
\hline & $\mathrm{HIV}^{-}$ & 0.0000 & 0.0047 & NS & \\
\hline
\end{tabular}




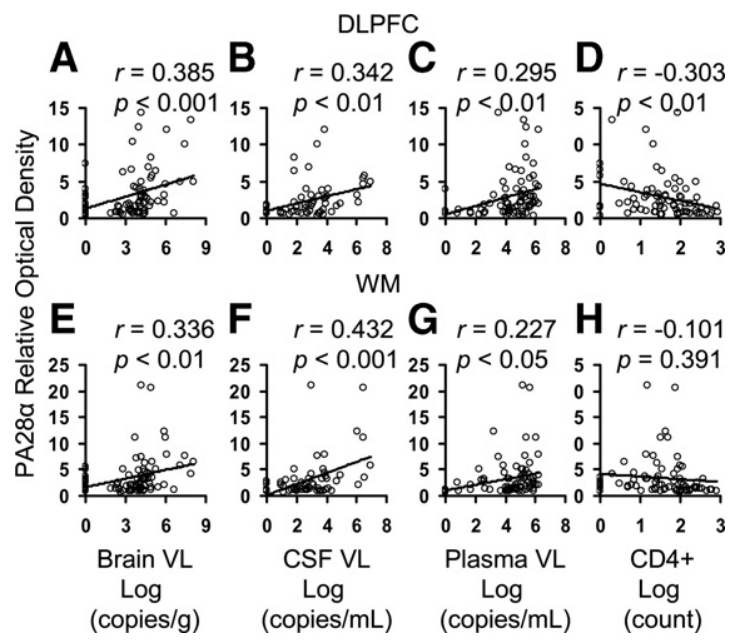

Figure 6. Immunoproteasome induction in DLPFC (A-D) and WM (E-H) was correlated significantly with virological and immunological status of HIV-infected subjects. HIV-1 RNA concentrations in brain (A and E), CSF (B and $\mathbf{F}$ ), and blood plasma (C and $\mathbf{G}$ ) were positively correlated with PA28 $\alpha$ levels. $\mathrm{CD}^{+}$lymphocyte counts in blood plasma were negatively correlated with PA28 $\alpha$ levels in DLPFC (D), but not WM $(\mathbf{H})$. Equivalent results for LMP7 were obtained (not illustrated). Statistics: Pearson's correlation.

ing Digit Symbol (Figure 5D) and Symbol Search (Figure $5 \mathrm{E})$, which reflect a decrease in the speed of information processing. The same relationship was not present in WM (not illustrated). No correlations between IPS levels and other functional domains evaluated by the National NeuroAIDS Tissue Consortium battery were observed (not illustrated).

\section{Correlation with Clinical Virology and Immunology}

Correlations between IPS subunit levels and VL in brain tissue, CSF, and plasma, and plasma CD4 ${ }^{+} \mathrm{T}$-cell count were assessed. DLPFC PA28 $\alpha$ levels were robustly and positively correlated with VL in all tissue compartments measured, and were negatively correlated with $\mathrm{CD}^{+}$ T-cell counts (Figure 6, A-D). WM PA28 $\alpha$ levels also correlated with VL (Figure 6, E-G) but were not correlated with $\mathrm{CD}^{+}{ }^{+} \mathrm{T}$-cell count (Figure $6 \mathrm{H}$ ). Analysis with LMP7 levels produced results equivalent to PA28 $\alpha$ (not illustrated). Assessment of IFN- $\gamma$ levels in brain tissue by enzyme-linked immunosorbent assay showed an increasing trend with $\mathrm{HIV}^{+}$subjects, but marked variability precluded further analysis (not shown).

\section{Localization in Neurons}

Glial and neuronal elements were positively stained for IPS subunits in HIVE. In general, immunoreactivity in white matter was more intense than in the cortex. Pathological structures characteristically present in HIVE were heavily stained. Microglia, macrophages, and oligodendrocyte nuclei were stained in microglial nodules and diffusely (Figure 7A). Neuronal cell bodies in neocortical laminae III and IV had positive staining for IPS subunits (Figure 7, B and D). WM axons often contained IPS
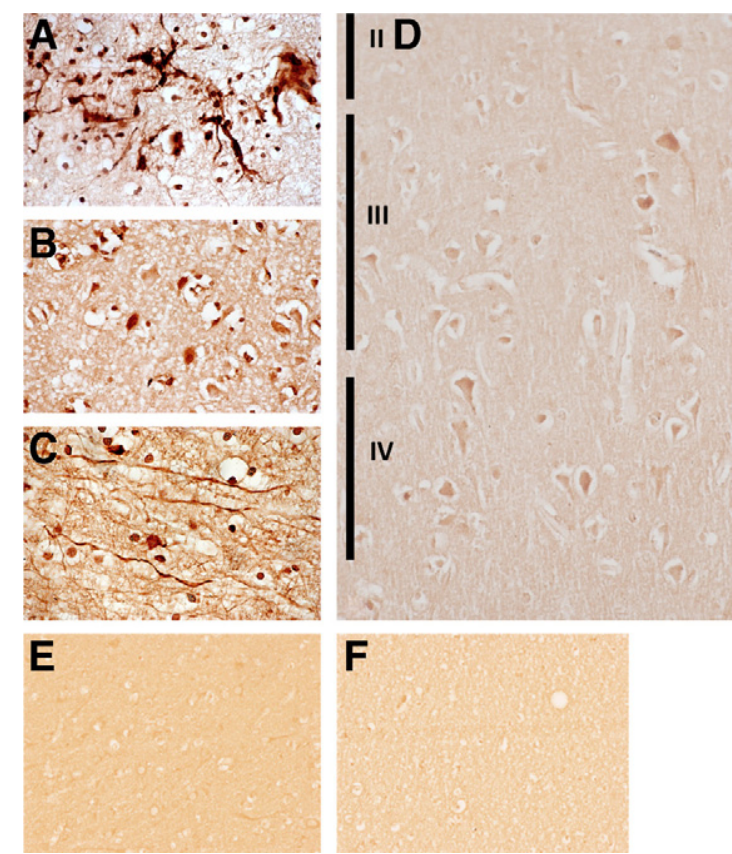

Figure 7. Immunoperoxidase histochemistry illustrates immunoproteasome subunit staining for PA28 $\alpha$ in glial and neuronal elements that are pathological in HIV encephalitis. Microglia, macrophages, and oligodendrocyte nucle are stained in a microglial nodule (A). Neuronal cell bodies in neocortical neurons were stained in focal pathology $(\mathbf{B})$ and were evident in laminae III and IV generally, though admittedly lighter than focal pathology (D). White matter axons often contained immunoproteasome subunits (C). Low immunoreactivity was observed in the (E) cortex and (F) white matter of HIV subjects.

subunits (Figure 7C). HIV $^{-}$sections of DLPFC (Figure 7E) and WM (Figure 7F) lacked focal immunostaining. Colocalization with neuronal markers (NeuN and neurofilament) was present in HIVE (Figure 8, A and B) but not $\mathrm{HIV}^{-}$sections (Figure 8 inserts). LMP2 was present primarily in perikaryal cytoplasm, whereas PA28 $\alpha$ was more evident in neuronal nuclei. LMP2 and PA28 $\alpha$ localized with neurofilament protein in WM axons. Dystrophic swollen axons, which are a neuropathological anomaly in HIVE, ${ }^{34-37}$ contained IPS (Figure 8B). Synaptic morphology is disturbed in HIVE, ${ }^{38}$ and these structures also contained IPS. Presynaptic boutons stained for synaptophysin showed the typical punctate staining pattern of synapses. Numerous synaptic boutons contained IPS (Figure 9A). Complete overlapping of IPS and synaptophysin suggested localization in presynaptic boutons (Figure 9B). Incomplete overlapping (Figure 9, C and D) suggested that IPS might be present in postsynaptic densities or synaptic astrocyte foot processes. Markers for glial cells including glial fibrillary acidic protein for hypertrophic astrocytes, CD68 for microglia/macrophages, and oligodendrocyte and myelin glycoprotein confirmed that IPS subunits were present in those cells as suggested in Figure 7 (not illustrated).

\section{Discussion}

Immunoproteasomes are induced widely in the brains of $\mathrm{HIV}^{+}$people compared with baseline levels in $\mathrm{HIV}^{-}$sub- 
A
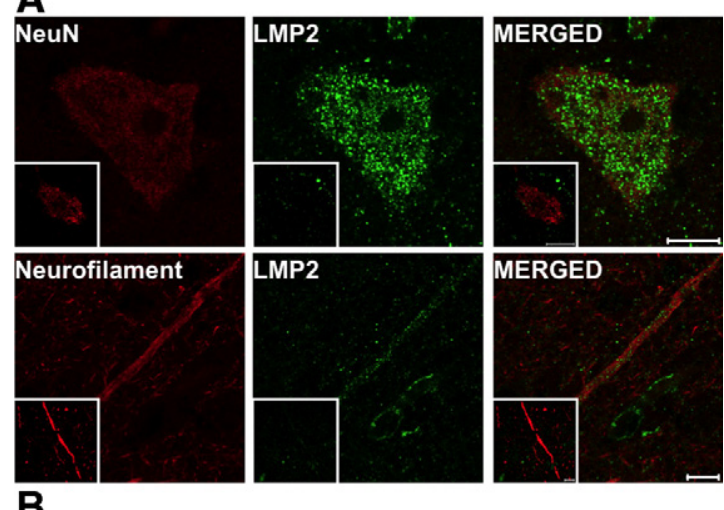

B

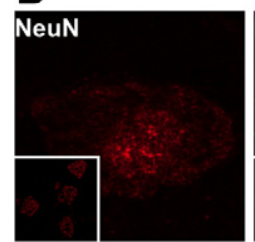

Neurofilament
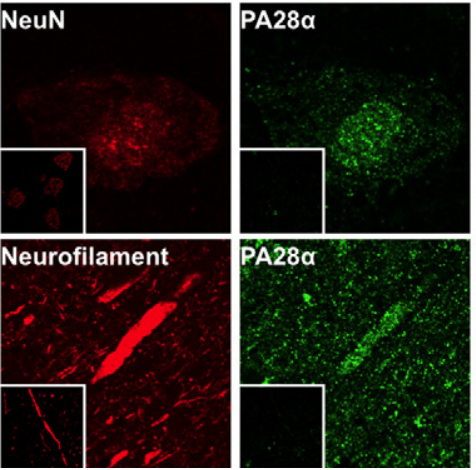

MERGED

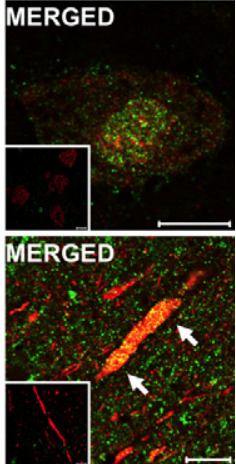

Figure 8. Immunoproteasome subunits expressed in neurons in HIV encephalitis. Dual indirect immunofluorescence staining for LMP2 (A) or PA28 $\alpha$ (B) shows colocalization with the neuronal markers NeuN and neurofilament in single optical sections from confocal microscopy. LMP2 is present in granular deposits in the parikaryon (A). PA28 $\alpha$ is more prominent in the nucleus (B). A pathologically swollen axon in white matter contains PA28 $\alpha$ (arrows in B), as do several other neurofilament-containing processes. Immunofluorescence stainings of $\mathrm{HIV}^{-}$sections (insets) reveal an absence of LMP2 and PA28 $\alpha$. Scale bar $=10 \mu \mathrm{m}$.

jects, and this abnormality is clinically and pathologically relevant. Induction of IPS with switching of 205 catalytic subunits and $11 \mathrm{~S}$ regulatory subunits of the CPS was present in about half of the $\mathrm{HIV}^{+}$decedents and pro-

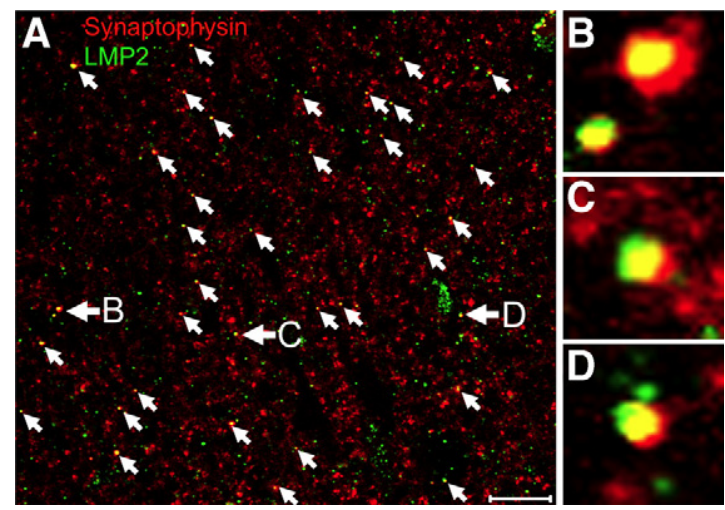

Figure 9. Immunofluorescence and laser confocal microscopy reveal that immunoproteasome subunit LMP2 is localized to some neocortical synapses in HIV encephalitis. A: LMP2 was colocalized within punctate deposits of synaptophy$\mathrm{sin}$, which is an established cell marker of presynaptic boutons (arrows). About $12 \%$ of labeled synapses in the figure contain immunoproteasome antigenicity. Complete overlapping of these two antigens was evident in some synapses and suggests that immunoproteasomes often are present in presynaptic boutons (B) Some synapses had incomplete overlapping (C and $\mathbf{D})$, which suggests that immunoproteasomes may be present in adjacent structures, including the postsynaptic density or in synaptic astrocyte foot processes. Scale bar $=10 \mu \mathrm{m}$. duced a three- to fourfold increase in IPS subunit concentration. IPS synthesis was relevant neuropathologically because it was significantly more prevalent in people with a postmortem diagnosis of HIVE, although HIVE was not required. The high prevalence of IPS induction in people with HIVE indicates that the neuropathologically evident inflammatory response to replicating HIV-1 in the brain is important, although it was not the sole risk factor. HIV-1 VL in brain tissue and CSF were correlated, as was HIV-1 VL in the plasma compartment. The latter observation agrees with studies that show linkage between HAD and abnormalities in the vascular compartment, including anemia and altered monocyte populations. ${ }^{1,39,40}$ A relationship between IPS induction and weakened systemic immunity was implied by the lower plasma CD4 ${ }^{+}$lymphocyte counts in affected subjects, and suggests that having advanced HIV/AIDS is a risk factor. ${ }^{1}$ The broad clinical-pathological impression that emerges from these results is that inflammatory defense against virus, ${ }^{15}$ systemically and in the brain, are involved in IPS induction. Nevertheless, HIVE was not required for IPS induction in the brain; other risk factors are involved.

IPS synthesis was significantly more prevalent in people with MCMD or HAD. IPS synthesis was present in the DLPFC and WM, although significant correlation with neurocognitive impairment was most evident in DLPFC. Indeed, IPS induction measured in the DLPFC was correlated specifically with performance on WCST-64 and Wechsler Adult Intelligence Scale III processing speed subtests, which are driven by circuits involved with executive function and speed of information processing located in Brodmann areas 9 and 46 of the DLPFC. ${ }^{32,33}$ IPS changes in WM were not correlated with these functional domains even though they were significantly different in infected people. The correlation between increased IPS in DLPFC and specific functional impairments driven by the DLPFC circuitry illustrates that region- and circuit-specific changes drive specific facets of the overall syndrome of HIV-associated neurocognitive decline (HAND). "Taskspecific" changes that correlate selectively with a change in gray matter versus white matter have been reported in HIV-1 infected people, and suggest that neocortical and subcortical pathologies both contribute to HAND. ${ }^{41}$

Authors of several reports have observed changes in constitutive proteasomes and immunoproteasomes associated with advanced age. 21,42,43 Potential influence of age on immunoproteasome induction associated with HIV infection was investigated by performing regression analysis with HIV status and age regressed on the quantified immunoproteasome subunits. The results indicated that DLPFC immunoproteasome subunit concentrations in $\mathrm{HIV}^{+}$subjects were not significantly influenced by age. WM subunit concentrations were associated with a slight decrease with increasing age of approximately 3\% per year. Thus, advanced age does not cause or contribute to the immunoproteasome induction, the three- to fourfold increase in immunoproteasome subunits, or the correlation between DLPFC immunoproteasome subunit concentrations and neurocognitive performance in $\mathrm{HIV}^{+}$ subjects. 
IFN- $\gamma$ mediated IPS induction in inflammatory cells and glia is consistent with a role in antigen presentation and host defense against virus infection. ${ }^{10,15}$ Our carefully documented observation that IPS synthesis occurs in neuronal perikarya, dystrophic axons, and synapses is emphasized because it is quite novel. The ability of neurons to undergo IPS induction is not widely appreciated $^{10,11,44}$ and could suggest that neurons may participate in self antigen presentation or are passively involved in a potentially harmful metabolic shift. Neuronal IPS induction has been observed in only two other neurodegenerative diseases, Huntington's disease and Alzheimer's disease. ${ }^{43,44}$ Neurons express IFN- $\gamma$ receptors and can synthesize Class I histocompatibility complexes in response to IFN- $\gamma,{ }^{43,44}$ which probably requires IPS induction. As well, exposure of neurons to IFN- $\gamma$ produces distinct neurophysiological changes in synaptic transmission pertaining to learning and memory. ${ }^{45}$ It is notable that correlation was present between HAND and IPS induction in brain cortex (predominantly neuronal), but not white matter (mostly glial). Thus, IPS synthesis in neurons could be particularly important for neocortical dysfunction due to HIV-1 infection. For example, synapses and dendrites are abnormal morphologically ${ }^{38}$ and biochemically 28,46 in HIVE and are potentially important targets for therapeutic intervention. ${ }^{47}$ Synapses and dendrites contain proteasomes and undergo localized protein turnover. ${ }^{18,19,48,49}$ Polyribosomes, mRNA, and the UPS all are present locally in synaptic and dendritic compartments, and they play a pivotal role in regulating synaptic function. ${ }^{19,49,50}$ Experimental clogging of protein turnover in these compartments can be especially devastating because proteins involved in memory formation in synapses and dendrites turn over with astonishing speed (minutes to hours). ${ }^{19}$ Local protein turnover in the synaptic compartment is necessary because synaptic transmission and memory formation require rapid local adjustments that require changes in protein synthesis and degradation. ${ }^{18}$ Blocking protein turnover at the level of synthesis in dendritic polyribosomes, or degradation via the UPS, alters the electrophysiological changes that drive learning and memory and impairs performance on tests of learning and memory. ${ }^{26,51}$

The accumulation of ubiquitinylated pathological protein is a virtual "signature" of pathological brain aging, reflecting unsuccessful removal of abnormal protein via the UPS. ${ }^{23-25,27}$ The slowing down of the UPS in normal and pathological brain aging is potentially damaging because effete and oxidatively damaged cellular proteins depend on UPS for efficient removal., ${ }^{9,21}$ Inherited defects of the UPS lead to Parkinson's disease and Angelman's syndrome. ${ }^{52,53}$ UPS protein processing is decreased sharply in Alzheimer's disease and during brain aging. ${ }^{20,21,54}$ As well, experimental disruption produces synaptic dysfunction and deficits in learning and memory. ${ }^{18-27,48,49}$ Likewise, the finding of increased accumulation of ubiquitin-stained deposits and high molecular weight ubiquitin conjugates in HIV/AIDS brains suggest UPS dysfunction. ${ }^{28}$ Neuronal autophagy, which aids in ubiquitin-protein aggregate degradation, is also reduced in HIV infection and may further exacerbate the accumu- lation of ubiquitinated protein conjugates due to UPS dysfunction. ${ }^{55,56}$

HAND represents a novel example of an acquired defect in the UPS linked to synaptic dysfunction. ${ }^{38,46}$ Though the proteasome subunit analysis does not examine the structure or quantity of fully assembled proteasome complexes, the HIV-associated increases in IPS and $11 \mathrm{~S}$ subunits implicate increasing integration of these subunits into newly formed IPS complexes at the expense of constitutively expressed counterparts, which are assembled four times slower than IPS. ${ }^{6,57,58}$ The replacement of constitutively expressed 19S regulator complexes on the proteasome core by the increased presence of $11 \mathrm{~S}$ activator complexes, which are integral to IPS formation and antigen processing, ${ }^{59,60}$ is important because $19 \mathrm{~S}$ processes many thousands of polyubiquitinylated protein species during routine turnover and cellular maintenance. After a folded protein has been tagged for destruction with polyubiquitin, it binds to a subunit of 19S. The 19S complex contains ATPases, chaperonins, unfoldases, and isopeptidases and caps the 20S core complex to form the 26S proteasome complex. The substrate is energized by ATPases, polyubiquitin is removed and recycled, and the polypeptide is unfolded and chaperoned "single file" into the $20 \mathrm{~S}$ core for hydrolysis. Virtually all folded proteins turn over by the $26 \mathrm{~S}$ proteasome in that manner. The $11 \mathrm{~S}$ activator, however, does not contain ATPases or isopeptidases. Thus, it can neither process folded polyubiquitinylated protein nor recycle ubiquitin monomers, which are absolutely essential for normal operation of the UPS. ${ }^{61-63}$ IPS induc-

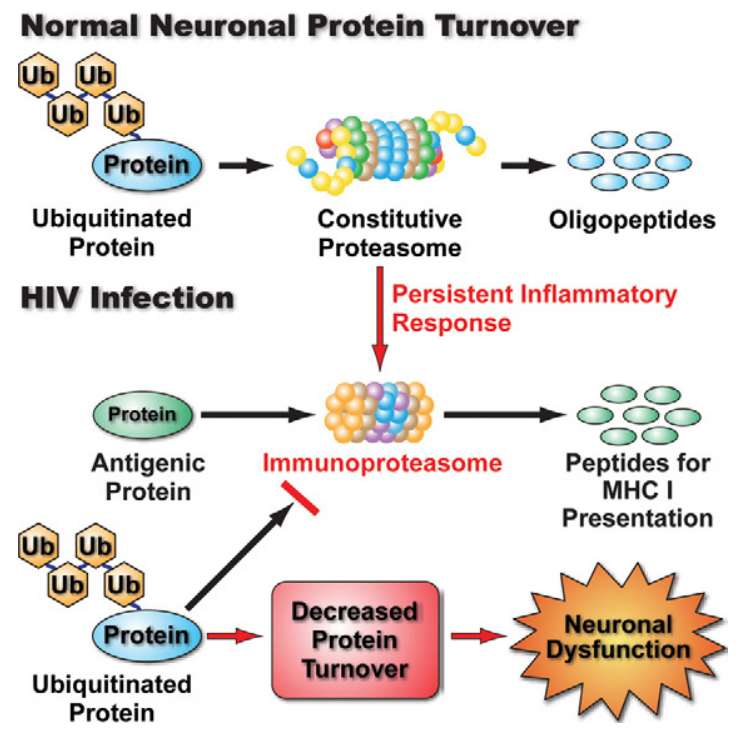

Figure 10. Persistent "hijacking" of brain proteasomes in HIV-1-infected people may lead to neuronal dysfunction. Normally, proteasome complexes rapidly turn over ubiquitinylated proteins. Persistent infection with HIV-1 produces inflammatory cytokines such as IFN- $\gamma$ that induce a temporary shift to immunoprotesome synthesis. In turn, the protein substrate repertoire is temporarily shifted toward the processing of unfolded peptides for class I antigen presentation. "Borrowing" the proteasome apparatus for heightened antigen presentation persists until the pathogen is eradicated, after which normal brain protein turnover should resume. Since HIV-1 infection is not eradicated in the brain, there is a persistent "hijacking" of the proteasome. Normal turnover of folded proteins is disrupted chronically. This leads eventually to the accumulation of pathologically misfolded proteins, neuronal dysfunction, and dementia. 
tion also coincides with destabilization and decrease in constitutive 26S proteasome complexes. ${ }^{12,64}$ The persistent replacement of 19S complexes on 20S complexes by increasing $11 \mathrm{~S}$ subunits due to HIV-1 infection is potentially devastating because thousands of cellular components can be perturbed by the changes in substrate repertoire..$^{9,12-14}$

Pervasive anomalies of the UPS in HAND suggest a novel concept regarding neuroinflammatory modulation of brain function (Figure 10). As 11S increasingly displaces 19S regulatory complexes, the neuronal proteasome population shifts to accommodate newly synthesized 11S/IPS complexes at the expense of the 26S proteasomes. The IPS complexes cannot perform "routine" turnover of polyubiquitinylated protein, which is the primary route for the turning over of most folded proteins. ${ }^{9}$ Instead, the IPS is specifically synthesized to process unfolded protein and small, unconjugated polypeptides with high efficiency. The best known function of the IPS is processing unfolded peptides for antigen presentation in Class I histocompatibility complexes. ${ }^{10,11}$ IFN- $\gamma$ induced increases of IPS synthesis reflect heightened immune surveillance in response to the presence of foreign antigen. ${ }^{15} \mathrm{~A}$ temporary "borrowing" of the UPS by IPS is tolerable physiologically because pathogens are normally eradicated in due time, and the IPS disappears quickly due to its inherent structural instability. ${ }^{7}$ The shift to IPS synthesis, however, is not transient in the HIV-1 infected brain because the CNS is a reservoir of persistently replicating HIV-1. ${ }^{16,17}$ HIV-1 provokes a long-lasting sustained cytokine response that includes tumor necrosis factor $\alpha$ and IFN- $\gamma$ expression, ${ }^{65,66}$ synthesis of inflammatory response protein ${ }^{28,67}$ and heightened expression of several IFN- $\gamma$ inducible genes. ${ }^{68}$ Instead of the typical "borrowing" of the UPS for immune defense, ${ }^{7}$ a persistent "hijacking" of the UPS characterizes aptly the nature of the changes in HIV-1-infected brain tissue.

\section{References}

1. McArthur JC, Brew BJ, Nath A: Neurological complications of HIV infection. Lancet Neurol 2005, 4:543-555

2. Kaul M, Garden GA, Lipton SA: Pathways to neuronal injury and apoptosis in HIV-associated dementia. Nature 2001, 410:988-994

3. Williams KC, Hickey WF: Central nervous system damage, monocytes and macrophages, and neurological disorders in AIDS. Annu Rev Neurosci 2002, 25:537-562

4. Budka $H$ : Neuropathology of human immunodeficiency virus infection. Brain Pathol 1991, 1:163-175

5. Wiley CA, Achim C: Human immunodeficiency virus encephalitis is the pathological correlate of dementia in acquired immunodeficiency syndrome. Ann Neurol 1994, 36:673-676

6. Aki M, Shimbara N, Takashina M, Akiyama K, Kagawa S, Tamura T, Tanahashi N, Yoshimura T, Tanaka K, Ichihara A: Interferon-gamma induces different subunit organizations and functional diversity of proteasomes. J Biochem 1994, 115:257-269

7. Heink S, Ludwig D, Kloetzel PM, Kruger E: IFN-gamma-induced immune adaptation of the proteasome system is an accelerated and transient response. Proc Natl Acad Sci USA 2005, 102:9241-9246

8. Raasi S, Schmidtke G, de Giuli R, Groettrup M: A ubiquitin-like protein which is synergistically inducible by interferon-gamma and tumor necrosis factor-alpha. Eur J Immunol 1999, 29:4030-4036

9. Glickman MH, Ciechanover A: The ubiquitin-proteasome proteolytic pathway: destruction for the sake of construction. Physiol Rev 2002, 82:373-428

10. Kloetzel PM: Generation of major histocompatibility complex class I antigens: functional interplay between proteasomes and TPPII. Nat Immunol 2004, 5:661-669

11. Kloetzel PM, Ossendorp F: Proteasome and peptidase function in MHC-class-I-mediated antigen presentation. Curr Opin Immunol 2004, 16:76-81

12. Bose S, Brooks P, Mason GG, Rivett AJ: Gamma-interferon decreases the level of $26 \mathrm{~S}$ proteasomes and changes the pattern of phosphorylation. Biochem J 2001, 353:291-297

13. Gaczynska M, Rock KL, Goldberg AL: Gamma-interferon and expression of MHC genes regulate peptide hydrolysis by proteasomes. Nature 1993, 365:264-267

14. Gaczynska M, Rock KL, Spies T, Goldberg AL: Peptidase activities of proteasomes are differentially regulated by the major histocompatibility complex-encoded genes for LMP2 and LMP7. Proc Natl Acad Sci USA 1994, 91:9213-9217

15. Tishon A, Lewicki H, Rall G, Von Herrath M, Oldstone MB: An essential role for type 1 interferon-gamma in terminating persistent viral infection. Virology 1995, 212:244-250

16. Langford D, Marquie-Beck J, de Almeida S, Lazzaretto D, Letendre S, Grant I, McCutchan JA, Masliah E, Ellis RJ: Relationship of antiretroviral treatment to postmortem brain tissue viral load in human immunodeficiency virus-infected patients. J Neurovirol 2006, 12:100-107

17. Kulkosky J, Bray S: HAART-persistent HIV-1 latent reservoirs: their origin, mechanisms of stability and potential strategies for eradication. Curr HIV Res 2006, 4:199-208

18. Hegde AN: Ubiquitin-proteasome-mediated local protein degradation and synaptic plasticity. Prog Neurobiol 2004, 73:311-357

19. Ehlers MD: Activity level controls postsynaptic composition and signaling via the ubiquitin-proteasome system. Nat Neurosci 2003, 6:231-242

20. Keller JN, Hanni KB, Markesbery WR: Impaired proteasome function in Alzheimer's disease. J Neurochem 2000, 75:436-439

21. Keller JN, Gee J, Ding Q: The proteasome in brain aging. Ageing Res Rev 2002, 1:279-293

22. Ma J, Wollmann R, Lindquist S: Neurotoxicity and neurodegeneration when PrP accumulates in the cytosol. Science 2002, 298:1781-1785

23. Tsuji T, Shimohama S: Protein degradation in Alzheimer's disease and aging of the brain. Prog Mol Subcell Biol 2002, 29:43-60

24. Chondrogianni N, Gonos ES: Proteasome dysfunction in mammalian aging: steps and factors involved. Exp Gerontol 2005, 40:931-938

25. Ciechanover A, Brundin P: The ubiquitin proteasome system in neurodegenerative diseases: sometimes the chicken, sometimes the egg. Neuron 2003, 40:427-446

26. Ding Q, Dimayuga E, Markesbery WR, Keller JN: Proteasome inhibition induces reversible impairments in protein synthesis. FASEB $J$ 2006, 20:1055-1063

27. Layfield R, Cavey JR, Lowe J: Role of ubiquitin-mediated proteolysis in the pathogenesis of neurodegenerative disorders. Ageing Res Rev 2003, 2:343-356

28. Gelman BB, Schuenke K: Brain aging in acquired immunodeficiency syndrome: increased ubiquitin-protein conjugate is correlated with decreased synaptic protein but not amyloid plaque accumulation. J Neurovirol 2004, 10:98-108

29. Morgello S, Gelman BB, Kozlowski PB, Vinters HV, Masliah E, Cornford M, Cavert W, Marra C, Grant I, Singer EJ: The National NeuroAIDS Tissue Consortium: a new paradigm in brain banking with an emphasis on infectious disease. Neuropathol Appl Neurobiol 2001, 27:326-335

30. Woods SP, Rippeth JD, Frol AB, Levy JK, Ryan E, Soukup VM, Hinkin CH, Lazzaretto D, Cherner M, Marcotte TD, Gelman BB, Morgello S, Singer EJ, Grant I, Heaton RK: Interrater reliability of clinical ratings and neurocognitive diagnoses in HIV. J Clin Exp Neuropsychol 2004, 26:759-778

31. Palmer S, Wiegand AP, Maldarelli F, Bazmi H, Mican JM, Polis M, Dewar RL, Planta A, Liu S, Metcalf JA, Mellors JW, Coffin JM: New real-time reverse transcriptase-initiated PCR assay with single-copy sensitivity for human immunodeficiency virus type 1 RNA in plasma. J Clin Microbiol 2003, 41:4531-4536

32. Anderson SW, Damasio H, Jones RD, Tranel D: Wisconsin Card Sorting Test performance as a measure of frontal lobe damage. J Clin Exp Neuropsychol 1991, 13:909-922

33. Monchi O, Petrides M, Petre V, Worsley K, Dagher A: Wisconsin Card 
Sorting revisited: distinct neural circuits participating in different stages of the task identified by event-related functional magnetic resonance imaging. J Neurosci 2001, 21:7733-7741

34. An SF, Giometto B, Groves M, Miller RF, Beckett AA, Gray F, Tavolato $B$, Scaravilli F: Axonal damage revealed by accumulation of beta-APP in HIV-positive individuals without AIDS. J Neuropathol Exp Neurol 1997, 56:1262-1268

35. Giometto B, An SF, Groves M, Scaravilli T, Geddes JF, Miller R, Tavolato B, Beckett AA, Scaravilli F: Accumulation of beta-amyloid precursor protein in HIV encephalitis: relationship with neuropsychological abnormalities. Ann Neurol 1997, 42:34-40

36. Raja F, Sherriff FE, Morris CS, Bridges LR, Esiri MM: Cerebral white matter damage in HIV infection demonstrated using beta-amyloid precursor protein immunoreactivity. Acta Neuropathol 1997, 93:184-189

37. Adle-Biassette $H$, Chretien F, Wingertsmann L, Hery C, Ereau T, Scaravilli F, Tardieu M, Gray F: Neuronal apoptosis does not correlate with dementia in HIV infection but is related to microglial activation and axonal damage. Neuropathol Appl Neurobiol 1999, 25:123-133

38. Masliah E, Heaton RK, Marcotte TD, Ellis RJ, Wiley CA, Mallory M, Achim CL, McCutchan JA, Nelson JA, Atkinson JH, Grant I: Dendritic injury is a pathological substrate for human immunodeficiency virusrelated cognitive disorders. HNRC Group the HIV Neurobehavioral Research Center. Ann Neurol 1997, 42:963-972

39. Gartner S: HIV infection and dementia. Science 2000, 287:602-604

40. Fischer-Smith T, Croul S, Sverstiuk AE, Capini C, L'Heureux D, Regulier EG, Richardson MW, Amini S, Morgello S, Khalili K, Rappaport J: CNS invasion by $\mathrm{CD}_{14}+/ \mathrm{CD} 16^{+}$peripheral blood-derived monocytes in HIV dementia: perivascular accumulation and reservoir of HIV infection. J Neurovirol 2001, 7:528-541

41. Gelman BB, Soukup VM, Holzer CE, 3rd, Fabian RH, Schuenke KW, Keherly MJ, Richey FJ, Lahart CJ: Potential role for white matter Iysosome expansion in HIV-associated dementia. J Acquir Immune Defic Syndr 2005, 39:422-425

42. Keller JN, Huang FF, Markesbery WR: Decreased levels of proteasome activity and proteasome expression in aging spinal cord. Neuroscience 2000, 98:149-156

43. Mishto M, Bellavista E, Santoro A, Stolzing A, Ligorio C, Nacmias B, Spazzafumo L, Chiappelli M, Licastro F, Sorbi S, Pession A, Ohm T, Grune T, Franceschi C: Immunoproteasome and LMP2 polymorphism in aged and Alzheimer's disease brains. Neurobiol Aging 2006 27:54-66

44. Diaz-Hernandez M, Hernandez F, Martin-Aparicio E, Gomez-Ramos P, Moran MA, Castano JG, Ferrer I, Avila J, Lucas JJ: Neuronal induction of the immunoproteasome in Huntington's disease. J Neurosci 2003, 23:11653-11661

45. Vikman KS, Owe-Larsson B, Brask J, Kristensson KS, Hill RH: Interferon-gamma-induced changes in synaptic activity and AMPA receptor clustering in hippocampal cultures. Brain Res 2001, 896:18-29

46. Gelman BB, Spencer JA, Holzer CE, 3rd, Soukup VM: Abnormal striatal dopaminergic synapses in National NeuroAIDS Tissue Consortium subjects with HIV encephalitis. J Neuroimmune Pharmacol 2006, 1:410-420

47. Bellizzi MJ, Lu SM, Gelbard HA: Protecting the synapse: evidence for a rational strategy to treat HIV-1 associated neurologic disease. J Neuroimmune Pharmacol 2006, 1:20-31

48. Bingol B, Schuman EM: Synaptic protein degradation by the ubiquitin proteasome system. Curr Opin Neurobiol 2005, 15:536-541

49. Yi JJ, Ehlers MD: Ubiquitin and protein turnover in synapse function. Neuron 2005, 47:629-632

50. Eberwine J, Belt B, Kacharmina JE, Miyashiro K: Analysis of subcel- lularly localized mRNAs using in situ hybridization, mRNA amplification, and expression profiling. Neurochem Res 2002, 27:1065-1077

51. Eisenberg M, Kobilo T, Berman DE, Dudai Y: Stability of retrieved memory: inverse correlation with trace dominance. Science 2003, 301:1102-1104

52. DiAntonio A, Haghighi AP, Portman SL, Lee JD, Amaranto AM, Goodman CS: Ubiquitination-dependent mechanisms regulate synaptic growth and function. Nature 2001, 412:449-452

53. Chung KK, Dawson VL, Dawson TM: The role of the ubiquitin-proteasomal pathway in Parkinson's disease and other neurodegenerative disorders. Trends Neurosci 2001, 24:S7-S14

54. Lam YA, Pickart CM, Alban A, Landon M, Jamieson C, Ramage R, Mayer RJ, Layfield R: Inhibition of the ubiquitin-proteasome system in Alzheimer's disease. Proc Natl Acad Sci USA 2000, 97:9902-9906

55. Alirezaei M, Kiosses WB, Flynn CT, Brady NR, Fox HS: Disruption of neuronal autophagy by infected microglia results in neurodegeneration. PLoS One 2008, 3:e2906

56. Zhu Y, Vergote D, Pardo C, Noorbakhsh F, McArthur JC, Hollenberg MD, Overall CM, Power C: CXCR3 activation by lentivirus infection suppresses neuronal autophagy: neuroprotective effects of antiretroviral therapy. FASEB J 2009, 23:2928-2941

57. Yewdell JW: Immunoproteasomes: regulating the regulator. Proc Nat Acad Sci USA 2005, 102:9089-9090

58. Griffin TA, Nandi D, Cruz M, Fehling HJ, Kaer LV, Monaco JJ, Colbert RA: Immunoproteasome assembly: cooperative incorporation of interferon gamma (IFN-gamma)-inducible subunits. J Exp Med 1998, 187:97-104

59. Groettrup M, Soza A, Eggers M, Kuehn L, Dick TP, Schild H, Rammensee HG, Koszinowski UH, Kloetzel PM: A role for the proteasome regulator PA28alpha in antigen presentation. Nature 1996, 381: 166-168

60. Preckel T, Fung-Leung WP, Cai Z, Vitiello A, Salter-Cid L, Winqvist O, Wolfe TG, Von Herrath M, Angulo A, Ghazal P, Lee JD, Fourie AM, Wu Y, Pang J, Ngo K, Peterson PA, Fruh K, Yang Y: Impaired immunoproteasome assembly and immune responses in PA28 ${ }^{-1-}$ mice. Science 1999, 286:2162-2165

61. Dubiel W, Pratt G, Ferrell K, Rechsteiner M: Purification of an $11 \mathrm{~S}$ regulator of the multicatalytic protease. J Biol Chem 1992, 267: 22369-22377

62. Hershko A, Ciechanover A: The ubiquitin system. Annu Rev Biochem 1998, 67:425-479

63. Ma CP, Slaughter CA, DeMartino GN: Identification, purification, and characterization of a protein activator (PA28) of the $20 \mathrm{~S}$ proteasome (macropain). J Biol Chem 1992, 267:10515-10523

64. Bose S, Stratford FL, Broadfoot KI, Mason GG, Rivett AJ: Phosphorylation of 20 S proteasome alpha subunit C8 (alpha7) stabilizes the $26 \mathrm{~S}$ proteasome and plays a role in the regulation of proteasome complexes by gamma-interferon. Biochem J 2004, 378:177-184

65. Shapshak P, Duncan R, Minagar A, Rodriguez de la Vega P, Stewart RV, Goodkin K: Elevated expression of IFN-gamma in the HIV-1 infected brain. Front Biosci 2004, 9:1073-1081

66. Griffin DE: Cytokines in the brain during viral infection: clues to HIV-associated dementia. J Clin Invest 1997, 100:2948-2951

67. Achim CL, Morey MK, Wiley CA: Expression of major histocompatibility complex and HIV antigens within the brains of AIDS patients. Aids 1991, 5:535-541

68. Masliah E, Roberts ES, Langford D, Everall I, Crews L, Adame A, Rockenstein E, Fox HS: Patterns of gene dysregulation in the frontal cortex of patients with HIV encephalitis. J Neuroimmunol 2004, 157:163-175 